\title{
FLARE COLOURS AND LUMINOSITIES
}

\author{
S. CRISTALDI* and M. RODONÖ** \\ Catania Astrophysical Observatory
}

\begin{abstract}
Flare colours determined from simultaneous $U B V$ observations made at Catania Observatory and from sequential $U B V$ observations made at McDonald Observatory are presented. They fit fairly well with the theoretical colours computed according to the Gurzadian's (1970) non-thermal model. Only part of the observed flare colours are consistent with the solar type models by Gershberg (1967) and Kunkel (1970).

From a $B$-band patrol of UV Cet-type stars carried out from 1967 to 1972, some quantitative estimates of flare frequencies and luminosities and of their average contribution to the stellar radiation are given.

The corresponding parameters for the Sun, which were estimated from 'white light' flare activity, are also given for comparison. The Sun and V $1216 \mathrm{Sgr}$ can be regarded as low-activity flare stars of the type found by Kunkel (1973).
\end{abstract}

\section{The Observations}

This paper presents some results on colours and luminosities of stellar flares which have been obtained from photo-electric observations made at Catania Observatory since 1967. Part of the observations have been published (Cristaldi and Rodonò 1970, 1973). Details on the instrumentation are given in the quoted references. Further data, which were obtained later, with the same equipment, are also included.

Moreover, the UV Cet flare colours included here were obtained by one of us (M.R.) from observations made at McDonald Observatory with the pulse counting photometer described by Nather and Warner (1971) and by Warner (1971) attached to a 91-cm Cassegrain reflector. An uncooled blue-sensitive photomultiplier tube RCA 4516 was used.

\section{The Flare Colours}

Table I gives some characteristics of the flare stars observed simultaneously in $U, B$ and $V$.

Flare colours have been computed according to the following expressions:

$$
\begin{aligned}
& (U-B)_{f}=-2.5 \psi \log \frac{\left(I_{f} / I_{*}\right)_{U}}{\left(I_{f} / I_{*}\right)_{B}}+(U-B)_{*} \\
& (B-V)_{f}=-2.5 \mu \log \frac{\left(I_{f} / I_{*}\right)_{B}}{\left(I_{f} / I_{*}\right)_{V}}+(B-V)_{*},
\end{aligned}
$$

where $\psi$ and $\mu$ are the colour transformation coefficients appropriate for the Catania natural system. $I_{\mathrm{f}}$ and $I_{*}$ are the observed deflections due to the flare excess radiation

* Institute of Physics, and

** Institute of Mathematics, University of Messina, Italy. 


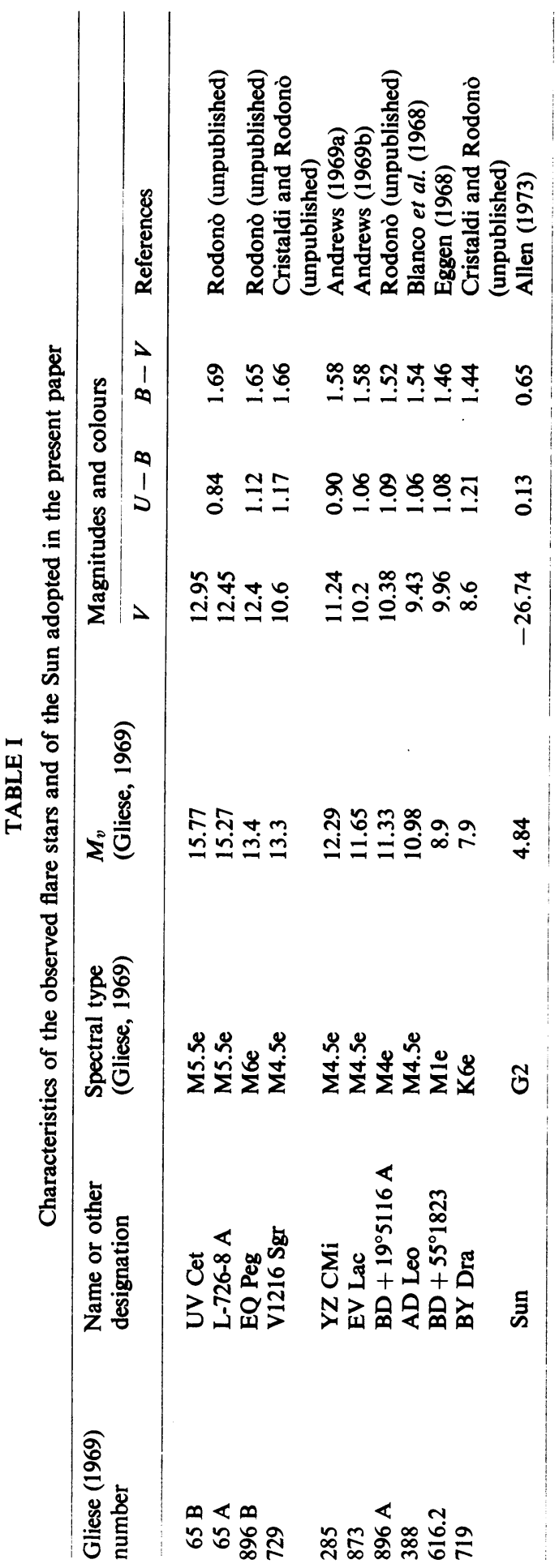




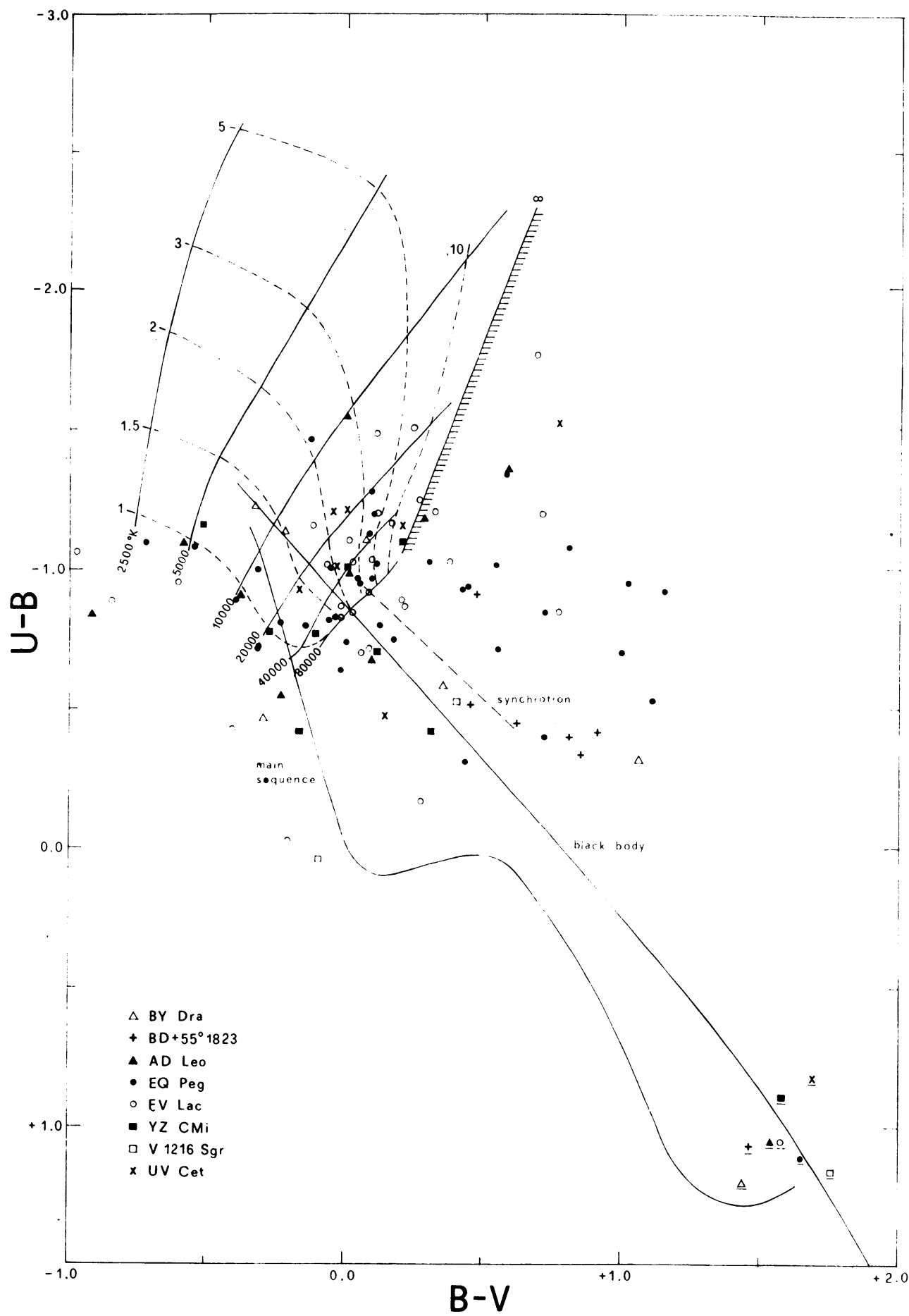

Fig. 1. The colours of the flare excess radiation at maximum. The main sequence, black body colours at different temperatures, synchrotron radiation colours with different spectral indices, and the Gershberg's (1967) flare grid model are drawn. The colours of the flare stars at minimum (underlined symbols) are on the lower right of the diagram. 


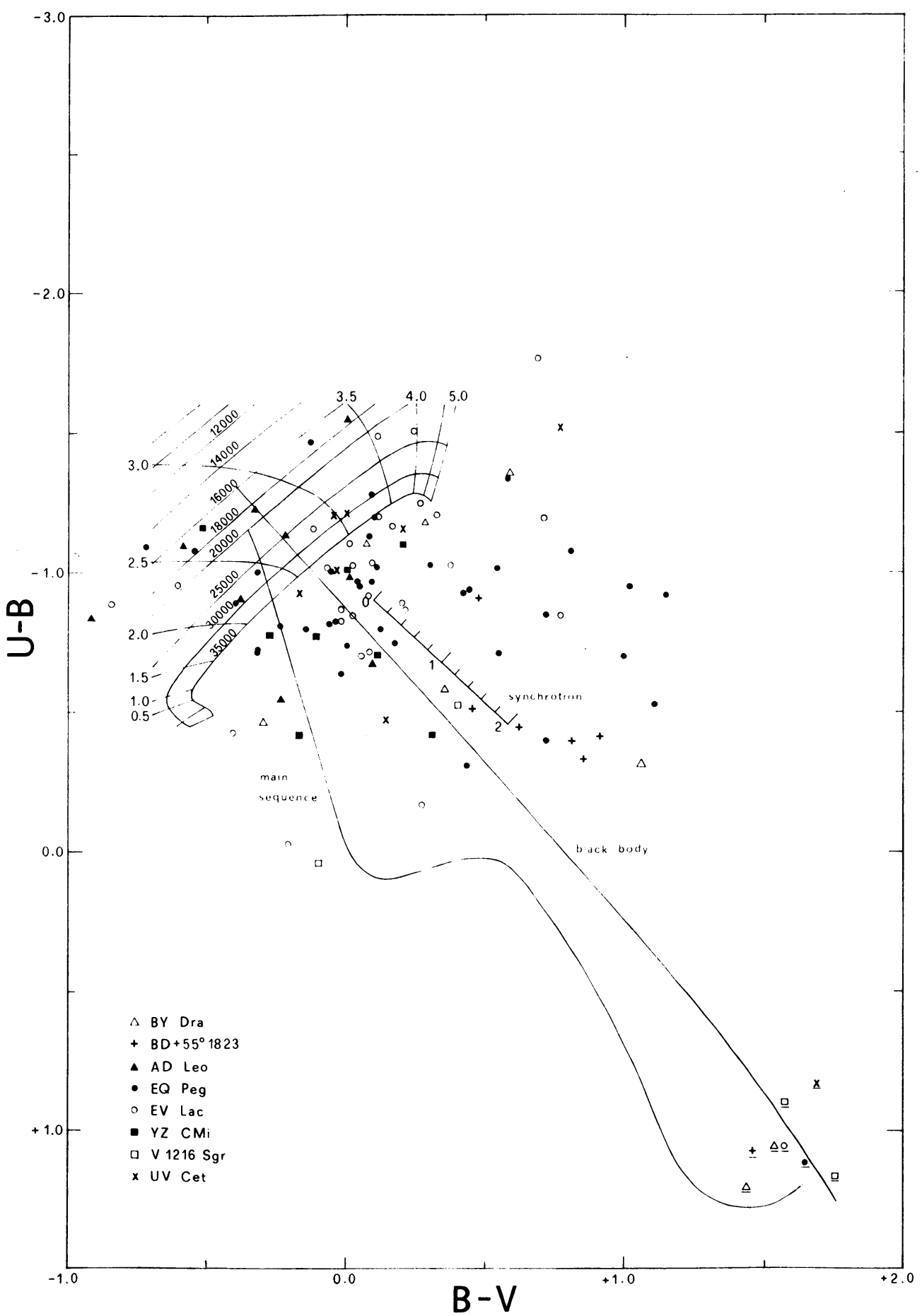

Fig. 2. The same quantities as in Figure 1, but the flare grid model by Kunkel (1970) is drawn. 


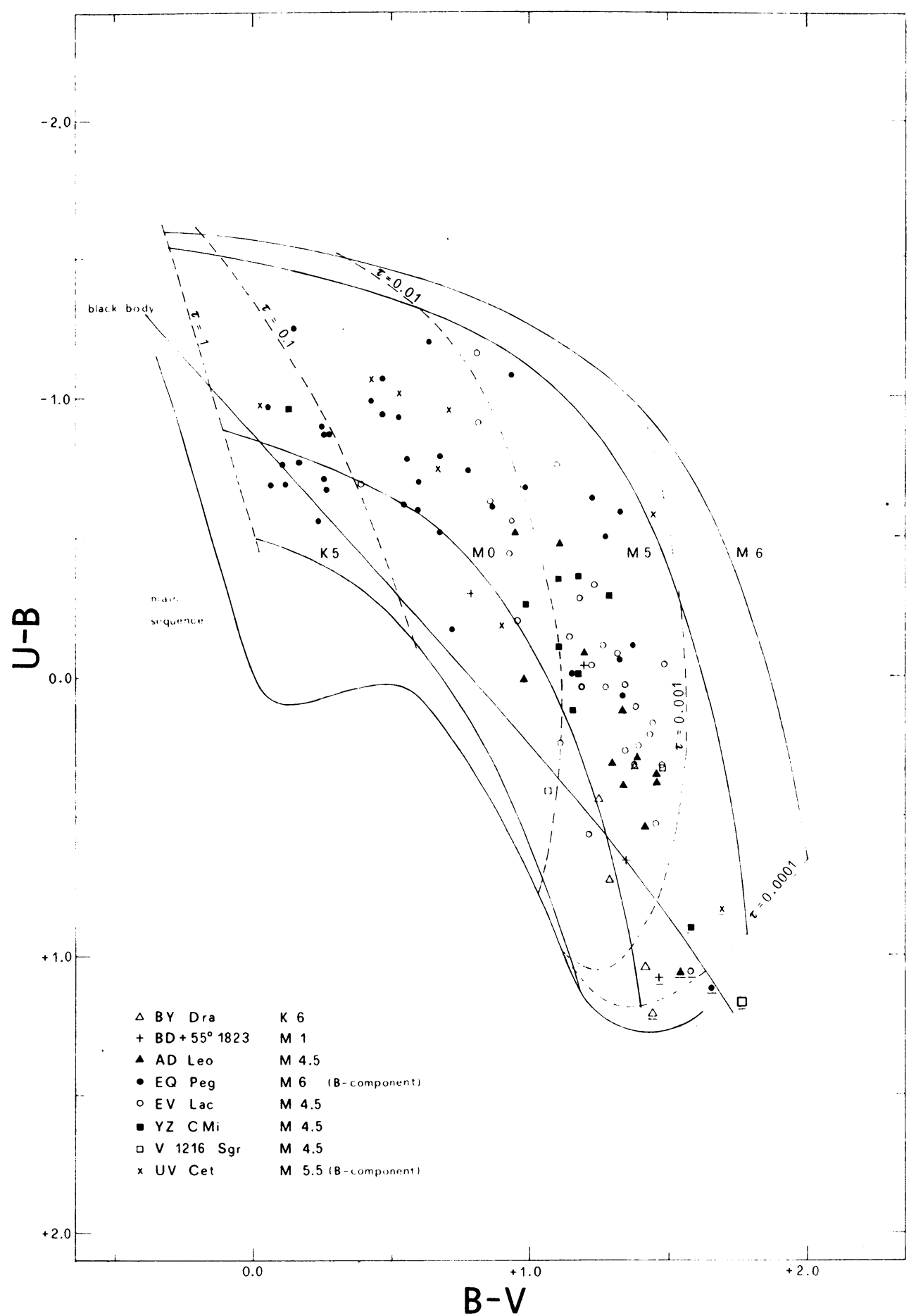

Fig. 3. The colours of flare stars at flare maximum. The main sequence, black body colours at different temperatures, and the flare grid model by Gurzadian (1972) are drawn. The EQ Peg and UV Cet flares are attributed to the fainter component of the binary systems $\mathrm{BD}+19^{\circ} 5116 \mathrm{AB}$ and $\mathrm{L}-726-8 \mathrm{AB}$. 


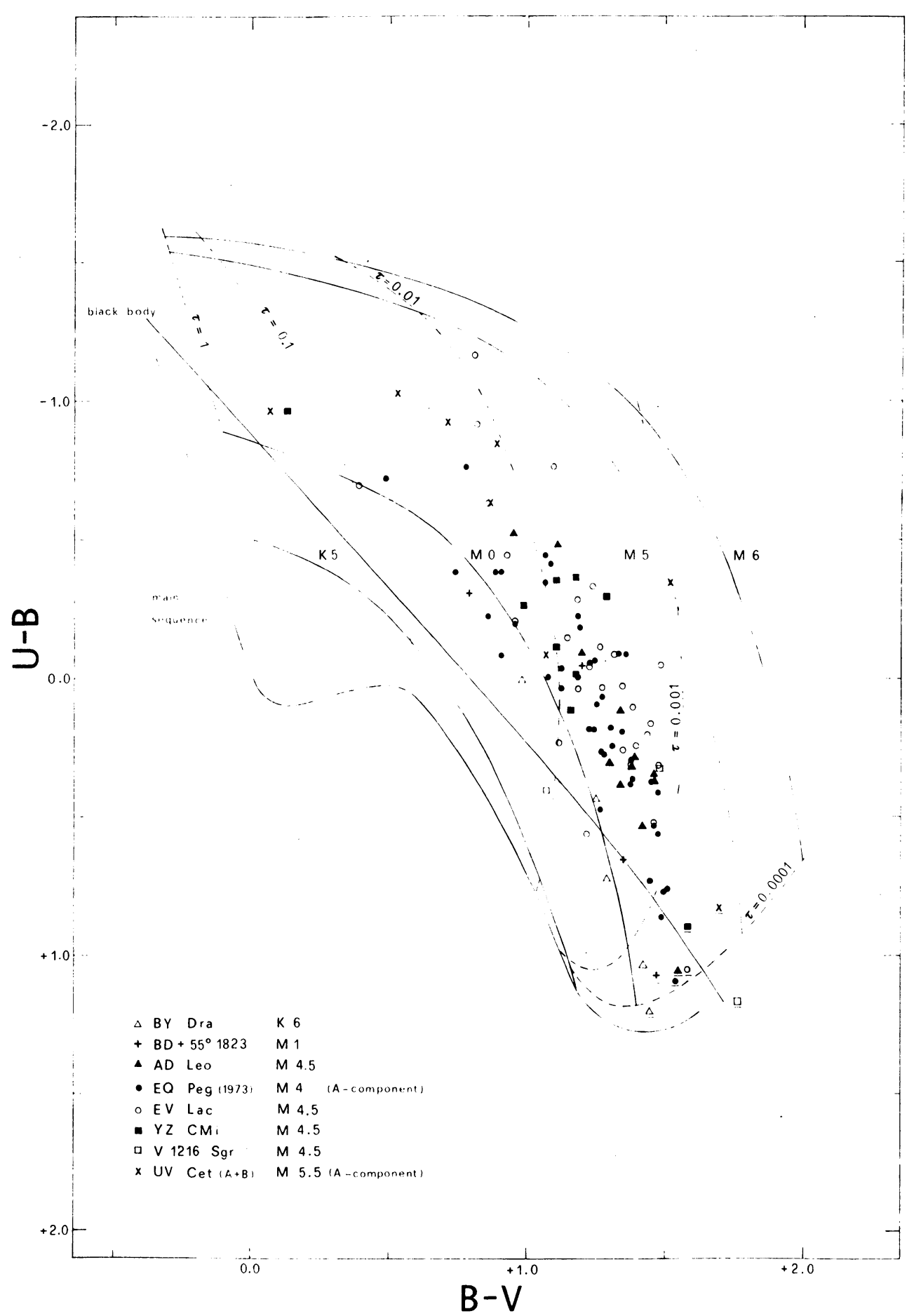

Fig. 4. The same quantities as in Figure 3, but the EQ Peg and UV Cet flares are attributed to the brighter component of the binary systems $\mathrm{BD}+19^{\circ} 5116 \mathrm{AB}$ and $\mathrm{L}-726-8 \mathrm{AB}$. 
and to the quiet star in the given colours, respectively, and $(U-B)_{*}$ and $(B-V)_{*}$ are the standard colours of the quiet star.

The colours obtained using the Equations (1) are those of the flare excess radiation only, without reference to the colours of the star to which the flare is attributed.

The colours of the excess radiation at flare maximum are plotted in the two colours $U-B, B-V$ diagram of Figure 1 . The colours of flare stars at minimum (underlined symbols) are in the lower right corner.

The main sequence, a line representing the black body colours at different temperatures and the grid model by Gershberg $(1964,1965,1967)$ are also drawn in Figure 1. Figure 2 is similar, but the grid model is drawn according to Kunkel (1970). Since faint fiares are not detectable in $V$-light the plotted colours refer to rather intense well observed flares.

Both the Gershberg hot - gas model and the Kunkel hydrogen - recombination model, are not adequate to account for the colours of all the flares observed. Due to the large colour discrepancy with Kunkel's model, corrections for the light contribution of a second flare component arising from the disturbed photosphere, as suggested by Kunkel (1970), were not applied.

Gurzadian $(1965,1972)$ has developed a non-thermal model according to which the optical stellar flares are produced by inverse-Compton effect and/or by nonthermal bremsstrahlung of fast electrons interacting with the photospheric photons. In Figure 3, the theoretical colour indices of the system 'star + Compton radiation' at flare maximum, are compared with the corresponding observed quantities:

$$
\begin{aligned}
& (U-B)_{*+f}=-2.5 \psi\left[\log \left(\frac{I_{f}+I_{*}}{I_{*}}\right)_{U}-\log \left(\frac{I_{f}+I_{*}}{I_{*}}\right)_{B}\right]+(U-B)_{*}, \\
& (B-V)_{*+f}=-2.5 \mu\left[\log \left(\frac{I_{f}+I_{*}}{I_{*}}\right)_{B}-\log \left(\frac{I_{f}+I_{*}}{I_{*}}\right)_{V}\right]+(B-V)_{*},
\end{aligned}
$$

where the symbols indicate the same quantities as in Equations (1). Equations (2) clearly show that the combined $(U-B)_{*+\mathrm{f}}$ and $(B-V)_{*+\mathrm{f}}$ colour indices depend on the colours of the star to which the flares are attributed. Therefore, for double flare stars such as EQ Peg and, probably, UV Cet too (see next section), we should know which component is flaring in order to correctly compare the observed and theoretical colours. Lacking this information, we considered both cases. The colours plotted in Figure 3 were computed attributing the observed flares to the fainter $B$-components of the systems containing EQ Peg and UV Cet, while those plotted in Figure 4 were computed attributing the observed flares to the brighter $A$-component. Also, in these figures, the main sequence and a line representing the black body colours at different temperatures are drawn.

All flare star colours at maximum of light obtained by Equations (2) fall within the model grid by Gurzadian. However, especially for faint flares, the combined 
colours of the unperturbed star plus the flare inverse Compton radiation are dominated by colours of the former at minimum, so that, this last test is less sensitive than the previous ones which refer to the colours of the flare excess radiation only. The predicted grouping of flare star colours along the particular line corresponding to the spectrum of the quiet star to which the flares are attributed is lacking; however, on the average, the additional flare radiation brings relatively red stars to the upper part and relatively blue stars to the lower part of the diagram, as predicted by Gurzadian's model. Moreover, we should like to point out that the location of the line representing the colours of a given star during flares, i.e. an equal spectrum line, depends on the assumed flare-star colours at minimum light. The colours, adopted by Gurzadian (1970) to obtain the theoretical grid model, are those of main sequence stars, which are not quite reliable for UV Cet type stars. So the grid would be somewhat lower on the left.

The scatter of points referring to EQ Peg flares is quiet large in Figure 3, mainly because some flares of the $A$-component have been attributed arbitrarily to the redder and about 2-magnitudes fainter $B$-component. The opposite is true for the plot in Figure 4, while in this case the scatter is obviously less noticeable. The same applies to the points representing UV Cet flares. However, the colours of both components being almost equal and their magnitudes differing by only $0 m 5$, the resulting spread of points due to erroneous identification of the flaring component is in this case never greater than $0^{m} \cdot 2$ both in $U-B$ and $B-V$.

Apparently the present simultaneous flare colour observations fit fairly well with Gurzadian's model, but the significance of this agreement is lessened by the relatively low sensitivity of Gurzadian's test, as quoted before. The solar type models by Gershberg and Kunkel are consistent with about $50 \%$ of the flare colours observed.

\section{The Flare Luminosities}

A photoelectric patrol of flare stars has been carried out at Catania Observatory since 1967. The $B$-band observations made up to 1972 are here considered. For each of the observed stars, the frequency distribution of $\log P_{B}$, where $P_{B}=\int\left(I_{\mathrm{f}} / I_{*}\right) \mathrm{d} t$ (in minutes) is the flare 'integrated intensity', is shown in Figure 5.

Each histogram is shifted vertically according to the absolute $B$ magnitude of the corresponding star and horizontally for an immediate reading, on the abscissa, of the logarithm of the total flare energy (in ergs) corresponding to a given $\log P_{B}$ class. The absolute calibration has been made assuming the $B$ luminosity and absolute magnitude of the Sun to be $1.95 \times 10^{33} \mathrm{erg} \mathrm{s}^{-1}$ and 5.48 respectively. For each histogram, the star name and spectral type, the total time coverage in hours and the number of the flares observed are given.

The frequency histogram for the Sun refers only to the so-called 'white-light' flares, i.e. flares during which the continuum enhancement is observed.

From Michard's (1959) flare sampling, we assume 18\% of chromospheric solar flares to be also 'white-light' flares. The average chromospheric flare frequency being 


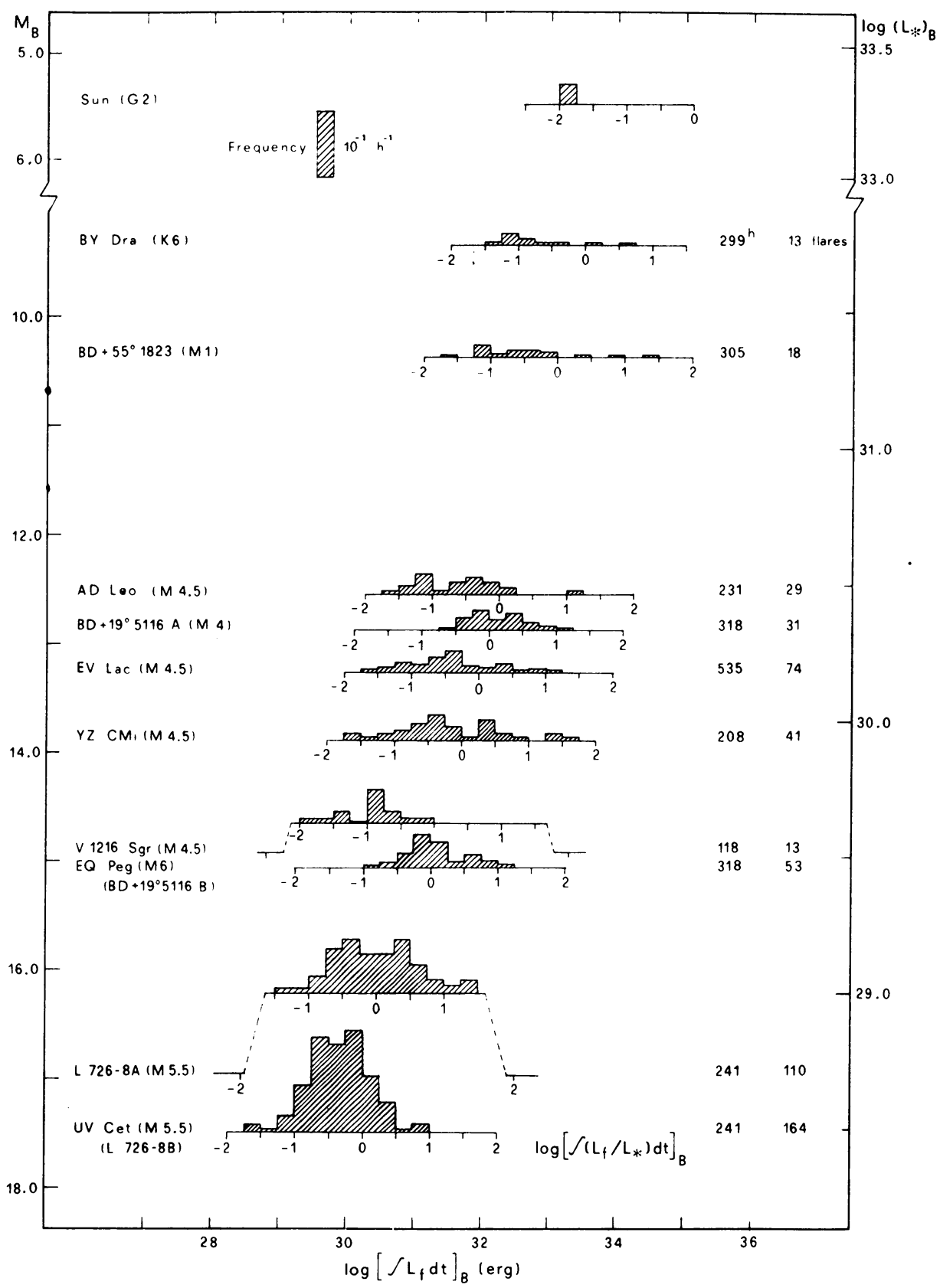

Fig. 5. Distribution of $\log P_{B}$ scaled to a system of absolute B-luminosity. The histogram for the Sun is confined to the class where the logarithm of the mean $P_{B}$ value for 'white light' flares falls. 


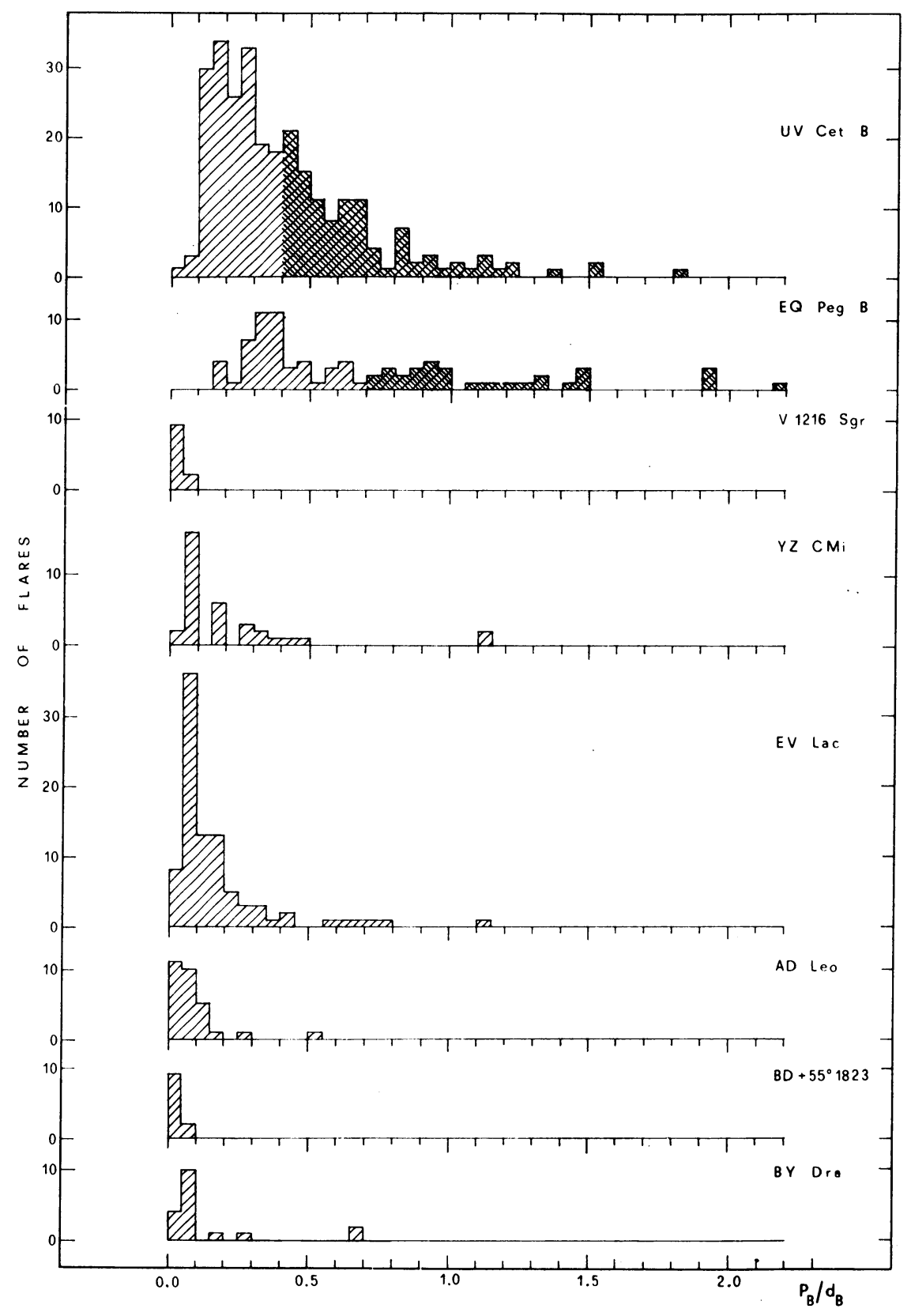

Fig. 6. Distribution of $P_{B} / d_{B}$, a dimensionless parameter which gives the mean excess luminosity during a flare normalized to the star luminosity at minimum. The UV Cet and EQ Peg flares are attributed to the fainter component of the respective binary system. Only for statistical purposes the flares on the heavily dashed region of each histogram are assumed as flares of the brighter components. 
$0.162 \mathrm{~h}^{-1}$ from 1937 to 1960 (Smith and Smith, 1963), we assume the 'white-light' flare frequency to be $0.029 \mathrm{~h}^{-1}$.

Assuming as typical parameters of 'white-light' solar flares (1) a continuum intensification of $150 \%$ of the undisturbed photosphere, (2) an extention of one thousandth of the solar disk and, (3) a duration of $10 \mathrm{~min}$, we obtain -1.8 for $\left(\log P_{B}\right)_{\text {Sun }}$. We do not have information about the distribution of 'white-light' solar flares with $P_{B}$, therefore the histogram presented in Figure 5 is confined to the class where the average $P_{B}$ value falls.

For the double flare stars EQ Peg and UV Cet, separate histograms for the individual components are given in Figure 5. The main criterion we adopted to attribute an observed flare to a given component is based on the value the parameter $P_{B} / d_{B}$ assumes for the given flare, $d_{B}$ being the total flare duration in the $B$-band. $P_{B} / d_{B}$ is a dimensionless parameter which indicates the mean excess energy release during the flare normalized to the luminosity of the quiet star. From our data (Figure 6) it results that, besides EQ Peg and UV Cet flares, the values of $P_{B} / d_{B}$ are lower than 0.5 for almost $90 \%$ of the flares observed, regardless of the $P_{B}$ values; i.e. the more powerful a flare the longer its duration. The large spread of values for EQ Peg and UV Cet can be interpreted as a computation effect arising from the overestimation of $P_{B}$ values when flares of the brighter component are attributed to the fainter one. As a matter of fact, the $P_{B}$ values are overestimated by almost one order of magnitude for the EQ Peg flares and by a factor of only 1.6 for the UV Cet ones.

EQ Peg is a well known double-flare star (Owen et al., 1972) whose fainter component contributes only about $60 \%$ to the observed flare frequency (Rodonò, 1973). Therefore, about $40 \%$ of flares due to the $A$-component have been erroneously attributed to the fainter $B$-component resulting in the quoted spread of $P_{B} / d_{B}$ values in Figure 6.

Although the brighter component of the visual binary L-726-8 is a good flare star candidate, no direct evidence has been yet collected. However, we interpret the large spread of $P_{B} / d_{B}$ values, which we obtain by attributing the observed flares to the fainter component (UV Cet), as an indirect evidence that the brighter component is also a flare star.

Therefore, the $P_{B} / d_{B}$ parameter offers an easy way to select flares of different components so that separate histograms for the individual components of these two binary systems were obtained. Of course, this can be done with confidence only for statistical purposes. The darker area, both for EQ Peg and UV Cet histograms in Figure 6 , includes $40 \%$ of the observed flares which we attribute to BD $+19^{\circ} 5116 \mathrm{~A}$ and L-726-8A, respectively.

An inspection of Figure 5 reveals that: (a) the total excess energy emitted in the $B$-band during a flare ranges from $3 \times 10^{28}$ to $3 \times 10^{34} \mathrm{erg}$ for stars whose $B$-luminosity ranges from about $3 \times 10^{28} \mathrm{erg} \mathrm{s}^{-1}$ to $2 \times 10^{33} \mathrm{erg} \mathrm{s}^{-1}$;

(b) the $\log P_{B}$ distributions flatten with increasing stellar luminosity, however, due to the detection threshold $\left(\approx-2.0\right.$ in $\left.\log P_{B}\right)$; the observed histograms, especially those for more luminous stars, could represent only the tails of the true distributions; 


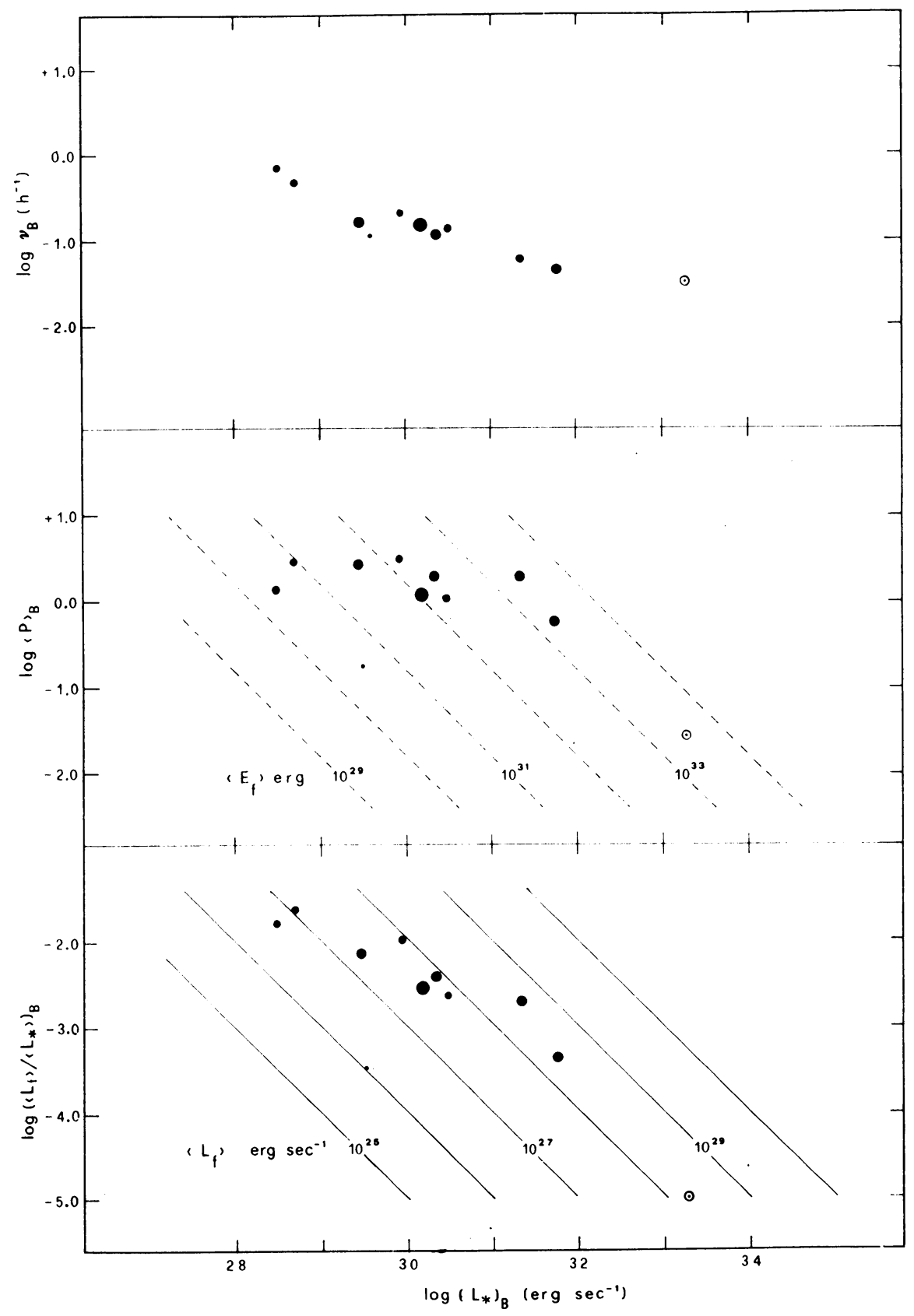

Fig. 7. The flare frequency $v_{B}$ (upper inset), the mean value of the excess energy during flares normalized to the energy emitted by the quiet star in one minute $P_{B}$ (middle inset), and the mean fractional contribution of flares to the stellar $B$-luminosity $\left.\left(\left\langle L_{\mathrm{Q}}\right\rangle\right\rangle^{*}\right)_{B}$ (lower inset) are plotted versus the stellar $B$-luminosity. The broken and the continuous diagonals calibrate the corresponding diagrams to absolute units. The dot diameters are proportional to the total patrol time. 
(c) the most powerful flares are observed in the most luminous stars, although these flares produce less conspicuous increases of the quiet luminosity;

(d) the maximum observed value of the total relative increase of $B$-band luminosity has never exceeded 60 times the energy emitted by the quiet star in one minute.

Some activity parameters obtained from our $B$-band observations vs. the star $B$-luminosity are presented in Figure 7 . The corresponding parameters for the Sun, which were obtained using the data already quoted, are also considered.

The upper inset of Figure 7 shows the well known fact that the observed flare frequency decreases as the stellar luminosity increases. If we assume that a given exponential distribution of the flare 'integrated intensity' $P_{B}$ similar to that given by Kunkel (1973) for $U$-amplitudes holds for any flare star, a linear decrease of $\log v_{B}$ with increasing $\log \left(L_{*}\right)_{B}$ should result. In fact, the flare visibility decreases as the stellar luminosity increases with a slope -1.075 . The relation observed is non-linear and, in any case, has a much lower slope. This requires that, the more luminous a star is, the higher the value to which the $\log P_{B}$ distribution extends. A similar trend was noticed by Kunkel (1973) for the peak amplitudes of flares. Moreover, anticipating a result presented below, the flare activity level could undergo a maximum at a given stellar luminosity. Therefore the expected decreasing of the flare activity, coupled with the continuous lowering of flare visibility, should produce a rapid drop of the observed flare frequency.

The middle inset of Figure 7 shows the trend of $\log \left\langle P_{B}\right\rangle$ versus $\log \left(L_{*}\right)_{B} ;\left\langle P_{B}\right\rangle$ being the mean value of the flare integrated energy for a given star. The set of parallel broken diagonals allows us to read directly from the diagram the average value of the energy $\left\langle E_{\mathrm{f}}\right\rangle$ emitted in the $B$-band during a flare. The plotted points show that on the average, the energy emitted during a flare equals the energy emitted by the quiet star in a few minutes, except for V1216 Sgr and for the Sun. V1216 Sgr and the Sun could be examples of the low active flare stars mentioned by Kunkel (1973).

For the observed stars, the absolute energy of a mean flare in $B$ light ranges from about $5 \times 10^{30}$ to $5 \times 10^{33} \mathrm{erg}$ in passing from the less luminous to the more luminous stars. Observational selection makes the latter value somewhat overestimated with respect to the former one.

The lower inset of Figure 7 shows the average observed contribution of flares to the star $B$-luminosity. The set of parallel continuous diagonals allows us to read the average luminosity of the star due to the observed flares. Observational selection is less severe in this case since (1) the main contribution to the energy radiated by the flare activity is due to the most powerful flares and (2) the total time coverage is rather extended for all stars presented.

The plotted points show that the energy emitted by flare activity becomes a progressively less important fraction of normal photospheric $B$-luminosity as the luminosity itself increases. The observed range is from more than $1 \%$ for stars with $M_{B} \approx 17$ to about $10^{-3} \%$ for the Sun.

The points referring to flare stars, except V1216 Sgr and the Sun, show a definite trend, with little spread, which could represent an 'activity sequence'. 
Two alternative conclusions can be drawn:

(a) assuming the Sun to belong to the above mentioned activity sequence and disregarding V1216 Sgr, we should conclude that the energy emitted by flare activity reaches a maximum $\left(\approx 9 \times 10^{28} \mathrm{erg} \mathrm{s}^{-1}\right)$ at about $\left(L_{*}\right)_{B}=4.5 \times 10^{32} \mathrm{erg} \mathrm{s}^{-1}$ for a star of spectral type near $\mathrm{K} 0$;

(b) the Sun and V1216 Sgr could define a lower activity sequence valid for relatively older stars. This agrees with the result obtained by Skumanich (1972) and by Blanco et al. (1974) in that the $\mathrm{K}$ emission line flux, which can be assumed as an activity indicator, decreases as the inverse square root of the star's age.

\section{Acknowledgements}

It is a pleasure to thank H. J. Smith, director of McDonald Observatory, for telescope time assignment during UV Cet 1972 international campaign, and G. Godoli, director of Catania Observatory, for helpful discussions.

\section{References}

Allen, C. W.: 1973, Astrophysical Quantities, 3rd. ed., The Athlone Press, London, p. 162. Andrews, A. D.: 1969a, Info. Bull. Var. Stars, No. 342.

Andrews, A. D.: 1969b, Info. Bull. Var. Stars, No. 370.

Blanco, C., Catalano, S., Marilli, E., and Rodonò, M.: 1974, Astron. Astrophys. 33, 257.

Blanco, V. M., Demers, S., Douglass, G. G., and FitzGerald, M. P.: 1968, Photoelectric Catalogue, U.S. Naval Obs. Publ. II Series 21.

Cristaldi, S. and Rodono, M.: 1970, Astron. Astrophys. Suppl. 2, 223.

Cristaldi, S. and Rodono, M.: 1973, Astron. Astrophys. Suppl. $10,47$.

Eggen, O. J.: 1968, Roy. Obs. Bull. 137.

Gershberg, R. E.: 1964, Izv. Krymsk. Astrofiz. Obs. 32, 133.

Gershberg, R. E.: 1965, Izv. Krymsk. Astrofiz. Obs. 33, 206.

Gershberg, R. E.: 1967, Astrofizika 3, 127.

Gliese, W.: 1969, Catalogue of Nearby Stars, Veröff. Astron. Rechen Inst. Heidelberg No. 22.

Gurzadian, G. A.: 1965, Astrofizika 1, 313.

Gurzadian, G. A.: 1970, Bol. Obs. Tonantzintla Tacubaya 5, 255.

Gurzadian, G. A.: 1972, Astron. Astrophys. $20,145$.

Kunkel, W.: 1970, Astrophys. J. 161, 503.

Kunkel, W.: 1973, Astrophys. J. Suppl. 25, 1.

Michard, R.: 1959, Ann. Astrophys. 22, 887.

Nather, R. E. and Warner, B.: 1971, Monthly Notices Roy. Astron. Soc. 152, 204.

Owen, F. N., Bopp, B. W., and Moffett, I. J.: 1972, Astrophys. Letters 10, 37.

Rodonò, M.: 1973, Boll. Soc. Ital. Fisica 99, 95.

Skumanich, R.: 1972, Astrophys. J. 171, 565.

Smith, H. J. and Smith, E. V. P.: 1963, Solar Flares, McMillan Co., New York, p. 71.

Warner, B.: 1971, in 'New Directions and New Frontiers in Variable Star Research', IAU Colloq. 15,

Veröff. Bamberg 9, 144.

\section{DISCUSSION}

$W$. Kunkel: What is the accuracy of the colours in the two-colour diagrams?

$M$. Rodono: About $\pm 0 m 2$ in $B-V$, and $\pm 0^{m} \cdot 3$ in $U-B$.

$W$. Kunkel: How many hours of monitoring are involved? 
M. Rodonò: About $200 \mathrm{~h} / \mathrm{star}$; exact numbers are in Figure 5.

$R$. E. Gershberg: Drs Cristaldi and Rodonò compared the three-colour flare observations with the nebular model and with Gurzadian's model (1970), but their preference for the nebular model is caused by using a different kind of comparison in these two cases. If the positions of the excited stars in the $(U-B),(B-V)$ plane for both models are computed, one obtains nearly the same values.

$M$. Rodono: We are aware of that. Unfortunately Dr Gurzadian's published model allows us to compute only the colours of the excited stars and not the colours of the flare excess radiation, as your model does.

Certainly, the $(U-B),(B-V)$ test is much more sensitive when applied to your model than to Gurzadian's one. 